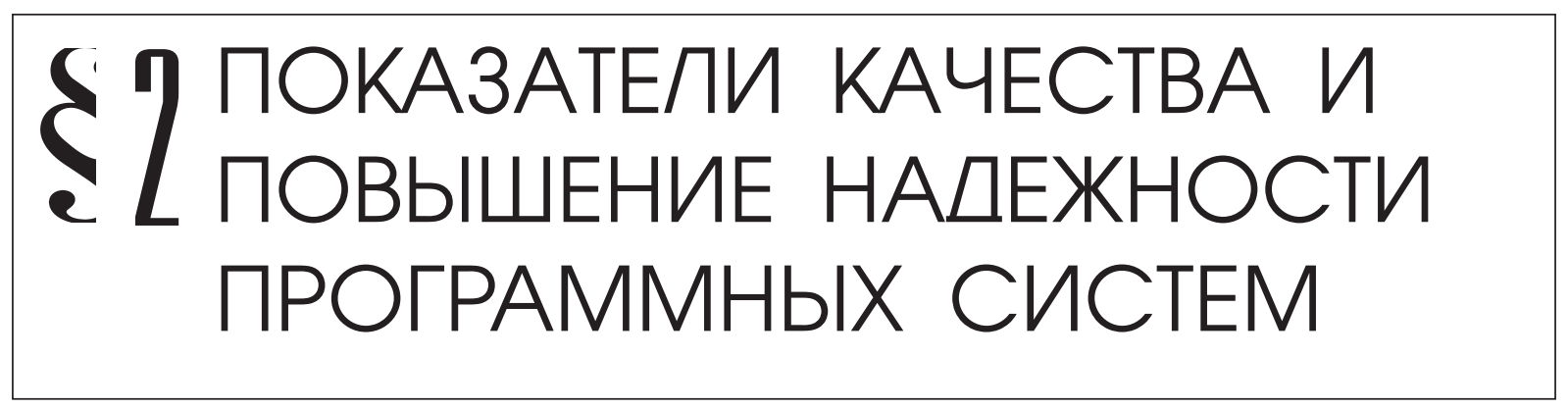

Поначугин А.В., Сокрута В.В.

\title{
СИСТЕМА ХРАНЕНИЯ ДАННЫХ КАК ФАКТОР ПОВЫШЕНИЯ ЭФФЕКТИВНОСТИ ФУНКЦИОНИРОВАНИЯ ИНФОРМАЦИОННЫХ СИСТЕМ
}

Аннотация: В данной статье рассматриваются пути улучшения эффрективности работы предприятия за счет увеличения скорости доступа к базам данных и общей отказоустойчивости системы. Изучаются характеристики систем хранения данных, такие как IOPS (количество операчий ввода-вывода в секунду) и разбираются виды устройств хранения данных и способы их подключения. Рассматриваются конкретные сценарии использования систем хранения данных относительно их способов соединения и других основныххарактеристик, применение их в предприятии. Дальнейшие пути расширения дискового пространства и действия при маситабировании вычислительной сети предприятия, с учетом появления новых гибридных систем хранения данных на основе флеш-памяти. В начале статьи рассматриваются виды хранения данных, $а$ также проводится сравнение СХД в зависимости от задач предприятия. Проводится исследование выбора способа соединения, изучение достоинств и недостатков каждого из них путем изучения технических характеристик и особенностей программных и annаратных систем хранения данных. Исследование влияние IOPS на быстродействие доступа к базам данных. Составлена таблица соотношения характеристикразличных систем хранения данных. В результате проведенной работы выявлены конкретные схемы применения СХД для небольщих и средних предприятий. Такой подход к задаче позволяет создать гибкое решение, которое не требует полную замену оборудования при увеличении требуемого дискового пространства и подключении дополнительных серверов, что в сумме дает большую экономическую и технологическую эффективность предприятию.

Ключевые слова: системы храненияданных, гипервизоры, жесткиедиски, твердотельные диски, дисковые массивы, сети хранения данных, отказоустойчивость, серверы, клиент- 
сервер, маситабируемость

Abstract: This article discusses ways to improve the performance of the enterprise by increasing the speed of access to data bases and overall system reliability. We study the characteristics of the data storage systems, such as IOPS (number of input-output operations per second) and understand types of storage devices and how they connect. Consider specific scenarios use storage on their process connections and other basic characteristics, their application in the enterprise. Further the expansion of space and action when scaled enterprise computing networks, taking into account the emergence of new hybrid storage solutions based on flash memory.At the beginning of this article discusses types of data storage, and storage is carried out a comparison based on the tasks of the enterprise. A study is under way to select the connection, the study of the advantages and disadvantages of each of them by studying the specifications and features of the software and hardware storage systems. A study on the impact of IOPS performance database access. A table of correlation characteristics of the various data storage systems.As a result of the work identified specific patterns of use of the storage system for small and medium-sized enterprises. This approach to the problem allows you to create a flexible solution that does not require the complete replacement of the equipment by increasing the required disk space and connect additional servers, for a total of more economic and technological efficiency of the enterprise.

Keywords: network storage, disk arrays, SSDs, hard disks, hypervisors, storage system, fault tolerance, servers, client-server, scalability

На сегодняшний день любая современная организация использует информационные технологии для того, чтобы совершенствовать свои методы работы [20, с.24-27].

Всё большую ценность для человека представляет актуальная информация. Ускоренное развитие технологий, темпа жизни и рост требований пользователей к контенту ставят перед нами вопрос, как и где хранить огромные массивы данных? Особенно актуальной эта проблема становится для современных крупных предприятий, так как деятельность всех их функциональных и обеспечивающих систем, неразрывно связаны с большим количеством информации, объём которой постоянно возрастает. В связи с этим появляется необходимость обеспечить не только надежное хранение информации, но и стабильный доступ к ней. Самым критичным моментом, особенно для систем с большим трафиком, является высокая доступность данных и выбор подходящего оборудования под определенные цели.

Сегодня в продаже существует большое количество разнообразных компьютерных систем, разных по стоимости и по набору выполняемых ими функций [18, с.75-79].

Ключевым вопросом как при проектировании информационной системы, так и при рассмотрении вариантов её масштабирования в данном случае является выбор технологии хранения данных. Для систем с высокой отказоустойчивостью и масштабируемостью возможно использование варианта с SAN (сеть хранения данных) или его упрощенной альтернативой, такой как NAS (англ. Network Attached Storage) и DAS (англ. Direct-attached storage). 
DAS - система хранения данных использующая прямое подключение, минуя сеть хранения данных, к примеру по Serial Attached SCSI или eSATA. Критическим недостатком использования DAS в корпоративных информационных системах - является невозможность разделять данные или неиспользуемые ресурсы с другими серверами. Единственное неоспоримое достоинства таких систем - простота настройки и подключение. Данное решение подходит для быстрого развёртывания в обеспечивающих подсистемах с небольшим трафиком, а также для меры экстренного реагирования в случае сбоя компонентов основной информационной системы.

NAS - подключается к сети с помощью кабеля Ethernet, и функционирует в качестве узла данной сети. Когда необходимо подключить клиента используется протокол SMB или NFS. Это позволяет разделять ресурсы с несколькими клиентами при работе с простыми файлами, но не имеет поддержки интеллектуальных функций, которые являются особенностью SAN (Storage Area Network). NAS не разработаны для высокой нагрузки и могут использоваться для простого обмена и долговременного хранения файлов, но не для серверного использования [12, с.55]. Что делает ограниченных возможность их использования в функциональных подсистемах корпоративной информационной системы предприятия.

Внедрение клиент-серверной архитектуры во второй половине 80-ых годов, способствовало увеличению объёмов, обрабатываемых и хранимых данных. Разработанная в то время технология SCSI (Small Computer System Interface) перестала удовлетворять растущим потребностям. Поэтому вскоре началась разработка нового протокола, целью которого являлось способность обойти ограничения SCSI. Так в 1994 году, вышла в свет первая редакция стандарта Fibre Channel (FC), ставшего впоследствии основой для построения сетей хранения данных (SAN) [16, с.16-27].

SAN появилась в середине 1990-тых годах, но из-за высокой стоимости внедрения была доступна только крупным IT-корпорациям. В настоящее время данная технология эффективного хранения данных доступна для любых, даже самых маленьких фирм [15, c.15-21].

О проблеме путей совершенствования систем хранения данных писал М.А. Сибиряков в своей работе «Способы повышения производительности систем хранения данных», где выводом было использование продвинутых компонентов кэш-памяти и продвинутые методы управления данными внутри неё [22, с. 1-3].

Исследование надежности настольных и Enterprise жестких дисков показывает Н.Е. Проскуряков, А.Ю. Ануфриева в работе «Анализ и перспективы современных систем хранения цифровых данных», где делается вывод что максимальную надежность можно получить используя именно Enterprise диски, так как количество отказов у них почти в два раза меньше по сравнению с обычными дисками [19, с.368-37 ].

В исследование «Распределенные системы хранения данных: анализ, классификация и выбор» Э.М. Мазур рассматривает необходимость тех или иных функций и механизмов в зависимости от задач бизнеса [17, с.33-60].

Для малого бизнеса SAN с высокой пропускной способностью, основанные на опти- 
ческих каналах всё еще слишком дороги. Но менее дорогие iSCSI сети хранения данных, которые используют Ethernet подключение, обычные сетевые карты и коммутаторы так же жизнеспособны и могут использоваться в небольших и средних компаниях.

Ключевая особенность SAN - Thin Provisioning, позволяет сообщать серверам любой объем дискового пространства, и наращивать его по мере надобности. Это позволяет избежать ненужной траты пространства на незагруженных серверах и более грамотно распределять дисковое пространство. Современные высокопроизводительные системы могут вмещать до 240 дисков SFF (Small Form Factor) или до 120 дисков LFF (Large Form Factor) что позволяет добиться высокой степени масштабируемости, по сравнению с классическими серверами, в которых 8-16 дисков максимально.

Отказоустойчивость является ключевым преимуществом сетей хранения данных, при выходе из строя одного сервера, второй может получить доступ к данным без промедления. Отказоустойчивость отдельной системы хранения обеспечивается дублированием всех основных компонентов, контролеров, блоков питания и даже процессорной части.

Встроенные RAID-контроллеры СХД на много надежнее и быстрее контролеров, которые используются в серверах за счет дублирования компонентов и большей кешпамяти [8, с.982].

Технология SAN позволяет хранить данные на нескольких серверах с большим уровнем эффективности и отказоустойчивости, по сравнению с традиционными дисковыми массивами. Так же может вдвое уменьшить количество дисков, что сэкономит деньги и электроэнергию, затраты на охлаждение систем.

Два основных способа соединения - Fiber Channel (оптический канал) и iSCSI [6, c.44]. Все они основаны на протоколе SCSI, но по своему его инкапсулируют. Если хост соединяется с SAN с использованием протокола iSCSI, то хост будет устанавливать эту связь с помощью стандартного сетевого адаптера, позволяет множеству компьютеров легко поддерживать соединение с одним устройством хранения данных [5, с.37]. Но все не так безоблачно.

Теоретически для сети хранения данных можно использовать уже существующую сеть предприятия, но не желательно, из-за большой нагрузки ISCSI протокол может перегрузить сеть и коммутаторы. В результате исследования были выявлены недостатки, к примеру сетевые карты 1Gb используемые на серверных материнских платах не предназначены для высокой нагрузки [7, с.67]. Это происходит из-за того, что сетевые адаптеры реализованы на обычных, не серверных чипах. Они порождают дополнительные накладные расходы по обслуживанию сетевого обмена, особенно в виртуализированных средах, что приводит к снижению производительности процессора, оперативной памяти до 30\%. [21, с.31-40].

Такие, встроенные сетевые адаптеры в высоконагруженных системах рекомендуется использовать для управления сервером, а для работы с дисками докупить дискретную карту на серверном чипе. Стоимость таких сетевых карт немного меньше по сравнению c Fiber Channel адаптерами. Самым выгодным способом использования такого соединения будет резервирование основного оптического соединения, для обеспечения от- 
казоустойчивости.

При оптоволоконном соединении сервера и SAN используется оптический хостадаптер, но в отличии от Ethernet адаптера оптоволоконное соединение не использует ТСР/ІР протокол. Достоинства такого соединения огромные скорости работы, до 16 Гигабит, и меньшая нагрузка на систему [19, с.99-105].

Но стоит учитывать, что SAN - это не только Fiber Channel, но и ISCSI. Очень часто эти две технологии используется параллельно. В частности, ISCSI дублирует Fiber Channel для надёжности, но при этом цена вопроса не возрастает в двое[3, с.87].

Для наглядности изученных данных проведены расчёты производительности классических дисковых подсистем и сравнить их характеристиками СХД.

Основные характеристики системы хранения данных это: количество операций ввода-вывода в секунду (IOPS) и отказоустойчивость.

Самая простая схема хранения данных в сервере это два SAS или SATA диска в RAID1[1, с.92] Таким образом достигается минимально возможное количество IOPS. SATA-диск на 7200 оборотов в секунду выдает около 100 IOPS, при использовании двух таких дисков в RAID1 можно рассчитать количество IOPS по схеме:

«(IOPS_диска * количество_дисков)/IO_пенальти»

В таблице 1 приведено значение пенальти для более часто используемых RAID конфигурации.

\begin{tabular}{|l|c|}
\hline \multicolumn{1}{|c|}{ RAID } & I/0 Пенальти \\
\hline RAID 0 & 1 \\
\hline RAID 1 & 2 \\
\hline RAID 5 & 4 \\
\hline RAID 6 & 6 \\
\hline RAID 10 & 2 \\
\hline
\end{tabular}

Таблица 1. Значения пенальти для более часто используемых RAID конфигурации

(200IOPS * 2)/2=200IOPS в случае SAS дисков и 100IOPS в случае SATA. Хранилище данных с характеристиками около 200IOPS будет достаточно лишь для небольшой базы размером в 300Мб и 2-4 пользователей. Таким образом (250IOPS * 4)/2=200IOPS RAID10 из четырех SAS дисков со скоростью вращения 15000 оборотов в секунду выдаст около 400-500IOPS, что должно быть достаточно для 10 пользователей небольшой базы [8].

Такие же цифры дадут расчёты производительности дисков сети хранения данных. Увеличение количества дисков в сервере не даст многократного увеличения производительности, так как ограничено количеством свободных слотов, до 8 дисков LFF и до 16 дисков SFF [2, с.133]. У СХД таких ограничений нет. Так же недостатком классических дисковых подсистем - отказоустойчивость осуществляется только за счет RAID контролера. Такой способ не подходит в ситуации с ростом пользователей [13, с.9-14].

Еще одним, более затратным путем решения, по сравнению с обычными дисками явля- 
ется установка серверных SSD - накопителей, (35000IOPS * 4)/2=70000IOPS дадут огромный прирост производительности. Недостатком таких решений является небольшой объем одного накопителя, и большая цена, в десятки раз превышающая классические HDD такого же объема. Проблема с отказоустойчивостью тоже лежит на контролере дисков.

Исследование затронуло гибридные системы хранения данных, обеспечивающие еще более высокую производительность, но более затратные. Гибридное хранилище - термин, используемый для описания систем хранения, основанные одновременно на твердотельных и обычных жестких дисках, это обеспечивает высокую производительность по доступной цене, доллар-за-IOPS (цена за операцию в секунду), и доллар-за-гигабайт. Это осуществляется автоматическим перемещением часто используемых блоков памяти на скоростной флеш-накопитель.

Повышение производительности кроме увеличения объема так же осуществляется за счет дедупликации и сжатия данных, хранящихся во флеш-памяти [4, с.596].

Узким местом является кеширование и запись информации на жесткие диски, различные производители используют свои методы для отбора часто используемых данных и записи измененной информации обратно на диск. Они сортируют и записывают данные на жесткие диски последовательно, так как скорость последовательной записи много быстрее случайной, так же позволяет избежать дефрагментации данных при чтении. Это позволяет использовать диски с меньшей скоростью вращения, и уменьшить количество передвижений считывающей головы. Это сказывается на потреблении электроэнергии, тепловыделении и ресурсам дисков. Второй компонент гибридной системы хранения - жесткие диски. Их функция - обеспечить наименьшую цену за гигабайт данных. Это осуществляется за счет дисков емкостью от 2Тб [11, с.93]. На рынке существует большое количество гибридных систем хранения под разные задачи, для большого и среднего бизнеса, с появлением новых технологий флеш-памяти стоимость таких решений будет уменьшаться, а также расти скорость доступа к данным.

На таблице 2 приведены соотношения важных характеристик SAN и классических схем хранения данных.

\begin{tabular}{|c|c|c|c|c|c|}
\hline Вариант & $\begin{array}{c}\text { Скорость } \\
\text { доступа }\end{array}$ & Объем & Надежность & Масштабируемость & Цена \\
\hline $\begin{array}{c}\text { RAID SAS } \\
\text { дисков }\end{array}$ & низкая & большой & низкая & незначительная & низкая \\
\hline $\begin{array}{c}\text { RAID SSD } \\
\text { дисков }\end{array}$ & высокая & малый & низкая & незначительная & высокая \\
\hline $\begin{array}{c}\text { SAN со } \\
\text { мнжеством } \\
\text { SAS }\end{array}$ & средняя & большой & высокая & значительная & средняя \\
\hline
\end{tabular}

Таблица 2. Соотношения важных характеристик SAN и классических схем хранения данных 
В результате исследования можно сделать вывод что для организаций, в зависимости от их размера и характера выполняемых ими задач, оптимально будет использовать различные подходы к построению систем хранения данных.

Для небольшой организации по соотношению цены и производительности, при использовании резервного копирования данных и небольших требованиях кдоступности данных возможно использовать обычные настольные компьютеры с высокопроизводительными процессорами и программным RAID, которые работает за счет чипсетов. Точкой отказа здесь может быть материнские платы и блоки питания, а также жесткие диски не Enterprise класса.

При высоких требованиях к надежности в небольшой организации будет лучшим выбором использовать серверные материнские платы и жесткие диски или уже готовые сервера, оснащенные надежными компонентами, что позволит увеличить время бесперебойной работы и свести количество точек отказа до минимума.

При стабильном росте количества сотрудников и размеров баз данных, стоит присмотреться к внешним сетевым хранилищам на основе iSCSI или SAS. Такие сетевые системы хранения данных, обладающие собственной гибкой файловой системой и интерфейсом конфигурации, понятным даже неподготовленному администратору, позволят увеличить скорость отклика базы данных и общую надежность системы. К тому же это позволит в будущем перейти на FC и использовать мощный кластер из нескольких серверов для балансировки нагрузки. Это решает все текущие и даже будущие проблемы инфраструктуры организации, например, в систему СХД возможно добавление дисков или подключение дополнительной дисковой полки, что позволит увеличить объем хранилища во много раз.

Минусом внедрения данного решения являются высокая цена данных и потраченное время на настройку, но они окупаются в ближайшем будущем за счет остальных преимуществ.

Хранилища SAN разнообразны и, благодаря большой конкуренции между производителями на рынке, появляется огромное количество решений, из которых даже самый требовательный заказчик выберет системы под свои нужды.

\section{Библиография :}

1. Blaum M., Brady J., Bruck J., and Menon J. Evenodd: An efficient scheme for tolerating double disk failures in RAID architectures // IEEE Transactions on Computing 44, 2 February 2011. $192 \mathrm{p}$.

2. Corbett P., English B., Goel A., Grcanac T., Kleiman S., Leong J., and Sankar, S. Row diagonal parity for double disk failure correction. //3rd Usenix Conference on File and Storage Technologies, San Francisco, CA, March 2014. 133 p.

3. Designing Storage Area Networks: A Practical Reference for Implementing Fibre Channel and IP SANs, Second Edition (Tom Clark, Addison-Wesley Professional, ISBN\# 0321136500), July 2011. 87 p.

4. Farley Marc. Building Storage Networks, second edition. The McGraw-Hill Companies, Inc., 2013. 596 p.

5. Fibre Channel Consultant: A Comprehensive Introduction (Robert Kembel and Roger Cummings, Northwest Learning Assoc. Inc., ISBN\# 0931836840), 2014. 37 p. 
6. Fibre Channel Consultant: Arbitrated Loop (Robert Kembel and Horst Truestedt, McGraw Hill, ISBN\# 0931836824), March 2011. $44 \mathrm{p}$.

7. IP SANS: An Introduction to iSCSI, iFCP, and FCIP Protocols for Storage Area Networks (Thomas Clark, Tom Clark, Addison-Wesley Professional, ISBN\# 0201752778), 2015. 67 p.

8. Jacob Bruce, Ng Spencer W., Wang David T. Memory Systems Cache, Dram, Disk. Morgan Kaufmann Publishers, 2008. $982 \mathrm{p}$.

9. Plank J.S. A new minimum density RAID code with a word size of eight // NCA-08: 7th IEEE International Symposium on Network Computing Applications, Cambridge, MA, July 2011. 182 p.

10. Plank, J. S. The RAID Liberation codes // FAST-20012:6th Usenix Conference on File and Storage Technologies (San Jose, February 2012). $97 \mathrm{p}$.

11. Storage Network Performance Analysis (Huseyin Simitci, Wiley, ISBN\# 076451685X), 2011.93 p.

12. Storage Security: Protecting, SANs, NAS and DAS (John Chirillo, et al, Wiley, ISBN\# 0764516884), 2014. 55 p.

13. Байкова И., Кулагин М. Современные дисковые системы RAID // Открытые системы. 2012. № 3. С. 9-14.

14. Васяева Е.С., Сибиряков М.А. Особенности развития высокопроизводительных систем хранения данных // сборник тезисов VIII Международной молодежной научной конференции» Научному прогрессу творчество молодых (2013). С. 56.

15. Ганьжа Д. Сеть устройств хранения // Журнал Сетевых Решений/LAN. 2001. № 2. С. 15-21.

16. Кузьминский М. Эволюция подсистемы ввода-вывода мэйнфреймов ІВМ. // Открытые системы. 1999. Январь. С. 16-27.

17. Мазур э.М. Распределенные системы хранения данных: анализ, классификация и выбор // Перспективы развития информационных технологий: сборник материалов XXVI Международной научно-практической конференции / Под общ. ред. С.С. Чернова. Новосибирск: Издательство ЦРНС, 2015. С. 33-60.

18. Поначугин А.В. Создание и перспективы открытых аппаратно-программных систем сетевого управления технологическими процессами // Информационные технологии в организации единого образовательного пространства. Сборник статей по материалам Международной научно-практической конференции преподавателей, студентов, аспирантов, соискателей и специалистов. Кафедра Прикладной информатики и информационных технологий в образовании. Нижний Новгород, 2015. С. 75-79.

19. Проскуряков Н.Е., Ануфриева А.Ю. Анализ и перспективы современных систем хранения цифровых данных // Известия ТулГУ. Технические науки. Вып. 3: Тула: Изд-во ТулГУ, 2013. С. 368-37.

20. Рыбакова А.С., Поначугин А.В. Информационные технологии: проблемы их внедрения, достоинства, недостатки // Актуальные проблемы гуманитарных и естественных наук. 2014. № 11-2. С. 24-27.

21. Сибиряков М.А., Васяева Е.С. Сравнительный анализ основных моделей интеллектуальных систем хранения данных в процессе их эволюции //Информационные технологии в профессиональной деятельности и научной работе. 2012. Ч 1. С. 99-105.

22. Сибиряков М.А. Способы повышения производительности систем хранения данных // Электронный научный журнал Института программных систем имени А.К. Айламазяна Российской академии наук, 2015. C. 1-3. 


\section{References:}

1. Blaum M., Brady J., Bruck J., and Menon J. Evenodd: An efficient scheme for tolerating double disk failures in RAID architectures // IEEE Transactions on Computing 44, 2 February 2011. 192 p.

2. Corbett P., English B., Goel A., Grcanac T., Kleiman S., Leong J., and Sankar, S. Row diagonal parity for double disk failure correction. //3rd Usenix Conference on File and Storage Technologies, San Francisco, CA, March 2014. 133 p.

3. Designing Storage Area Networks: A Practical Reference for Implementing Fibre Channel and IP SANs, Second Edition (Tom Clark, Addison-Wesley Professional, ISBN\# 0321136500), July 2011.87 p.

4. Farley Marc. Building Storage Networks, second edition. The McGraw-Hill Companies, Inc., 2013.596 p.

5. Fibre Channel Consultant: A Comprehensive Introduction (Robert Kembel and Roger Cummings, Northwest Learning Assoc. Inc., ISBN\# 0931836840), 2014. 37 p.

6. Fibre Channel Consultant: Arbitrated Loop (Robert Kembel and Horst Truestedt, McGraw Hill, ISBN\# 0931836824), March 2011. $44 \mathrm{p}$.

7. IP SANS: An Introduction to iSCSI, iFCP, and FCIP Protocols for Storage Area Networks (Thomas Clark, Tom Clark, Addison-Wesley Professional, ISBN\# 0201752778), 2015. 67 p.

8. Jacob Bruce, Ng Spencer W., Wang David T. Memory Systems Cache, Dram, Disk. Morgan Kaufmann Publishers, 2008. $982 \mathrm{p}$.

9. Plank J.S. A new minimum density RAID code with a word size of eight // NCA-08: 7th IEEE International Symposium on Network Computing Applications, Cambridge, MA, July 2011. 182 p.

10. Plank, J. S. The RAID Liberation codes // FAST-20012:6th Usenix Conference on File and Storage Technologies (San Jose, February 2012). 97 p.

11. Storage Network Performance Analysis (Huseyin Simitci, Wiley, ISBN\# 076451685X), 2011.93 p.

12. Storage Security: Protecting, SANs, NAS and DAS (John Chirillo, et al, Wiley, ISBN\# 0764516884), 2014. 55 p.

13. Baikova I., Kulagin M. Sovremennye diskovye sistemy RAID // Otkrytye sistemy. 2012. № 3. S. 9-14.

14. Vasyaeva E.S., Sibiryakov M.A. Osobennosti razvitiya vysokoproizvoditel'nykh sistem khraneniya dannykh // sbornik tezisov VIII Mezhdunarodnoi molodezhnoi nauchnoi konferentsii» Nauchnomu progressu tvorchestvo molodykh (2013). S. 56.

15. Gan'zha D. Set' ustroistv khraneniya // Zhurnal Setevykh Reshenii/LAN. 2001. № 2. S. 15-21.

16. Kuz'minskii M. Evolyutsiya podsistemy vvoda-vyvoda meinfreimov IBM. // Otkrytye sistemy. 1999. Yanvar'. S. 16-27.

17. Mazur E.M. Raspredelennye sistemy khraneniya dannykh: analiz, klassifikatsiya i vybor // Perspektivy razvitiya informatsionnykh tekhnologii: sbornik materialov KhXVI Mezhdunarodnoi nauchno-prakticheskoi konferentsii / Pod obshch. red. S.S. Chernova. Novosibirsk: Izdatel'stvo TsRNS, 2015. S. 33-60.

18. Ponachugin A.V. Sozdanie i perspektivy otkrytykh apparatno-programmnykh sistem setevogo upravleniya tekhnologicheskimi protsessami // Informatsionnye tekhnologii v organizatsii edinogo obrazovatel'nogo prostranstva. Sbornik statei po materialam Mezhdunarodnoi nauchno-prakticheskoi konferentsii prepodavatelei, studentov, aspirantov, soiskatelei i spetsialistov. Kafedra Prikladnoi informatiki i informatsionnykh tekhnologii v obrazovanii. Nizhnii Novgorod, 2015. S. 75-79.

19. Proskuryakov N.E., Anufrieva A.Yu. Analiz i perspektivy sovremennykh sistem khraneniya tsifrovykh dannykh // Izvestiya TuIGU. Tekhnicheskie nauki. Vyp. 3: Tula: Izd-vo TulGU, 2013. S. 368-37. 
20. Rybakova A.S., Ponachugin A.V. Informatsionnye tekhnologii: problemy ikh vnedreniya, dostoinstva, nedostatki // Aktual'nye problemy gumanitarnykh i estestvennykh nauk. 2014. № 11-2. S. 24-27.

21. Sibiryakov M.A., Vasyaeva E.S. Sravnitel'nyi analiz osnovnykh modelei intellektual'nykh sistem khraneniya dannykh v protsesse ikh evolyutsii //Informatsionnye tekhnologii v professional'noi deyatel'nosti i nauchnoi rabote. 2012. Ch 1.S. 99-105.

22. Sibiryakov M.A. Sposoby povysheniya proizvoditel'nosti sistem khraneniya dannykh // Elektronnyi nauchnyi zhurnal Instituta programmnykh sistem imeni A.K. Ailamazyana Rossiiskoi akademii nauk, 2015. S. 1-3. 